\title{
Decreased Incidence of Oncology Admissions in 75 Helios Hospitals in Germany during the COVID-19 Pandemic
}

\author{
Peter Reichardt $^{\mathrm{a}} \quad$ Andreas Bollmann $^{\mathrm{b}}$ Sven Hohenstein ${ }^{\mathrm{b}} \quad$ Bertram Glass $^{\mathrm{a}}$ \\ Michael Untch ${ }^{a}$ Annette Reichardt ${ }^{c}$ Daniel Amrein ${ }^{a}$ Ralf Kuhlen ${ }^{d}$ \\ ${ }^{a}$ Department of Oncology and Palliative Care, Helios Klinikum Berlin-Buch, Berlin, Germany; \\ ${ }^{b}$ Heart Center Leipzig and Leipzig Heart Institute, Leipzig, Germany; ${ }^{\mathrm{C} B e r l i n}$ Cancer Institute, Berlin, Germany; \\ ${ }^{\mathrm{d}}$ Helios Health, Berlin, Germany
}

\section{Keywords}

COVID-19 $\cdot$ Helios $\cdot$ Oncology admissions

\begin{abstract}
Background: The COVID-19 pandemic lead to a massive shutdown of social life in Germany starting in March 2020. Elective medical treatment was substantially reduced but urgent diagnostics and treatment including cancer care should not have been affected. Materials and Methods: We analyzed the number of oncology admissions to 75 German Helios hospitals during 2 time periods in 2020 and compared the data with the respective periods in 2019. The study included nearly 69,000 admissions in total. Results: A highly significant reduction in overall cancer admissions was seen for the early lockdown period from 13 March to 28 April 2020 compared to the same period in 2019. After an official communication advising the health system to return to normal practice on 29 April 2020 , we again found a highly significant difference in admissions compared to the respective time in the previous year. Subgroup analysis shows a significant impact of age $>75$ years, high hospital volume, and intermediate or high COVID-19 case volume in the federal states. Gender had no impact on admission numbers. The effects and significance levels were comparable in nearly all different diagnostic subgroups according to the ICD codes. Conclusions: For cancer diagnosis and treatment, we found a statistically significant decrease in hospital admissions in the range of $10-20 \%$ for both study periods in comparison to the previous year.
\end{abstract}

(c) 2020 S. Karger AG, Basel (c) 2020 S. Karger AG, Basel

www.karger.com/ort

Karger $\stackrel{2}{*}$

\section{Introduction}

Due to the COVID-19 pandemic, a near-total shutdown of social life was brought into effect in Germany on 13 March 2020 (the early lockdown period). Visits to GPs and hospital admissions were restricted to urgent treatment only. Some diagnostic procedures and treatments were postponed for several months. The legal advice clearly stated that cancer diagnosis and treatment should not be affected by these measures.

On 29 April 2020, an official communication advised the health system to return to normal practice (the late recovery period).

In order to assess the impact of the shutdown on cancer diagnosis and treatment, we analyzed the number of hospital admissions with any cancer diagnosis within our hospital network during the 2 target periods (the early lockdown period and the late recovery period) in comparison to the same periods in the previous year, 2019.

Helios Healthcare is the largest network of hospitals in Germany and Europe. Every year, we treat $>5$ million patients in 86 acute care Helios hospitals and 10 prevention centers across Germany.

\section{Method}

Data from 75 Helios hospitals involved in cancer diagnosis and treatment were analyzed. The remaining 11 Helios hospitals are not involved in cancer care and were therefore excluded from the analysis. The study periods were defined as 13 March to 28 April 
Table 1. Total number of cancer patients

\begin{tabular}{|c|c|c|c|c|c|c|}
\hline & \multicolumn{3}{|c|}{ Early period } & \multicolumn{3}{|c|}{ Late period } \\
\hline & $\begin{array}{l}\text { study } \\
\text { cohort }\end{array}$ & $\begin{array}{l}\text { control } \\
\text { cohort }\end{array}$ & $\begin{array}{l}\text { incidence rate } \\
\text { ratio }(95 \% \mathrm{CI})\end{array}$ & $\begin{array}{l}\text { study } \\
\text { cohort }\end{array}$ & $\begin{array}{l}\text { control } \\
\text { cohort }\end{array}$ & $\begin{array}{l}\text { incidence rate } \\
\text { ratio }(95 \% \mathrm{CI})\end{array}$ \\
\hline Total admissions per day & 345.7 & 400.3 & $0.86(0.85-0.88)^{*}$ & 332.1 & 386.9 & $0.86(0.84-0.88)^{*}$ \\
\hline \multicolumn{7}{|l|}{ Age } \\
\hline$\leq 64$ years & 146.6 & 163.8 & $0.90(0.87-0.92)^{*}$ & 133.1 & 154.0 & $0.86(0.84-0.89)^{*}$ \\
\hline 65-74 years & 95.5 & 106.7 & $0.90(0.86-0.93)^{*}$ & 93.6 & 103.9 & $0.90(0.87-0.94)^{*}$ \\
\hline$\geq 75$ years & 103.5 & 129.8 & $0.80(0.77-0.83)^{*}$ & 105.4 & 129.0 & $0.82(0.79-0.85)^{*}$ \\
\hline \multicolumn{7}{|l|}{ Gender } \\
\hline Male & 184.4 & 210.6 & $0.88(0.85-0.90)^{*}$ & 175.8 & 206.7 & $0.85(0.83-0.88)^{*}$ \\
\hline Female & 161.2 & 189.7 & $0.85(0.82-0.88)^{*}$ & 156.3 & 180.2 & $0.87(0.84-0.90)^{*}$ \\
\hline \multicolumn{7}{|l|}{ Hospital volume } \\
\hline Low & 7.0 & 10.2 & $0.69(0.60-0.79)^{*}$ & 8.4 & 11.5 & $0.73(0.64-0.83)^{*}$ \\
\hline Intermediate & 38.3 & 47.0 & $0.82(0.77-0.87)^{*}$ & 40.2 & 48.1 & $0.84(0.79-0.89)^{*}$ \\
\hline High & 300.3 & 343.0 & $0.88(0.86-0.90)^{*}$ & 283.5 & 327.2 & $0.87(0.85-0.89)^{*}$ \\
\hline \multicolumn{7}{|l|}{ COVID-19 case volume } \\
\hline Low & 100.5 & 107.4 & $0.94(0.90-0.97)^{*}$ & 98.0 & 107.5 & $0.91(0.88-0.95)^{*}$ \\
\hline Intermediate & 151.7 & 177.0 & $0.86(0.83-0.88)^{*}$ & 144.0 & 167.1 & $0.86(0.83-0.89)^{*}$ \\
\hline High & 93.5 & 115.8 & $0.81(0.78-0.84)^{*}$ & 90.1 & 112.3 & $0.80(0.77-0.84)^{*}$ \\
\hline
\end{tabular}

CI, confidence interval. Asterisks denote significance.

2020 (early lockdown period) and 29 April to 14 June 2020 (late recovery period) compared to the same periods in 2019. Patients with a main diagnosis of the ICD codes C15-C26, C30-C39, C40C41, C45-C49, C50, C51-C58, C60-C63, C64-C68, and C81-C96 were included in the analysis.

\section{Hospital Volume}

The hospital volume was categorized with respect to the number of admissions per hospital during the control period. We computed tertiles as low $\leq 96$, intermediate 97-293, and high $>293$.

\section{Volume of COVID-19 Cases in German Federal States}

For the German federal states in which the hospitals are located, we generated statistics based on COVID-19 cases occurring up to the last date of the study period (14 June 2020). Out of 16 federal states, Helios runs hospitals in 13, which are represented in this study.

The COVID-19 case volume was categorized with respect to the relative number of cases per federal state. Again, tertiles were computed, resulting in low $\leq 133.3$, intermediate $>133.3$ and $\leq 206.4$, and high $>206.4$.

The information on COVID-19 cases was obtained from the Robert Koch Institute. The numbers of inhabitants in the German federal states were obtained from Statistisches Bundesamt and are based on census data from 31 December 2018.

\section{Statistical Analysis}

Administrative data were extracted from QlikView (QlikTech, Radnor, Pennsylvania, USA). Incidence rates (IR) for Helios hospital admissions were calculated by dividing the number of cumulative admissions by the number of days in each time period. Incidence-rate ratios (IRR) comparing the study periods to each of the control periods or the early study period to the late study period were calculated using Poisson regression to model the number of hospitalizations per day. We calculated the number of admissions for all combinations of factor levels (i.e., age, sex, hospital volume, COVID-19 case volume), hospitals, and admission dates (of the corresponding period). These frequencies were used to create the dependent variables of the statistical models.

Inferential statistics were based on generalized linear mixed models (GLMM), specifying hospitals as the random factor $[1,2]$. We employed Poisson GLMMs with log link function for count data. Effects were estimated with the lme4 package v1.1-21 [3] in the $R$ Environment for Statistical Computing v3.6.1 (64-bit build) [4]. In all models, we specified varying intercepts for the random factor. The IRR values for the different factor levels were based on different models comparing the periods. Additionally, we employed another model for each factor with the variables period, treatment contrasts for the factor levels (for comparisons with the level at baseline), and the corresponding interactions. We report incidence-rate ratios (calculated by exponentiation of the negative of the regression coefficients) together with $95 \%$ confidence intervals (CIs, for the comparisons of the 2 periods) and $p$ values (for the interactions). For all tests, we applied a 2 -tailed $5 \%$ error criterion for significance.

\section{Results}

Table 1 shows the results for the total number of cancer patients. We compared the numbers in the study cohort with those in the control cohort for the early and the late period, respectively, and found a highly significant decrease in hospital admissions in both periods. We analyzed several subgroups (age, gender, hospital volume, and COVID-19 case volume) separately for the early and late periods, and confirmed a significant difference between study cohort and control cohort in all of these subgroups. This shows that the impact of the lockdown was independent of age, gender, the size of the respective hos- 
Table 2. Groups of cancer diagnosis

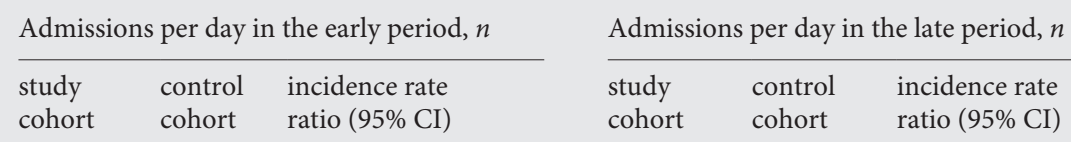

Malignant neoplasms of the digestive organs (ICD C15-C26), number of hospitals: 73, number of admissions: 14,496

\begin{tabular}{llllllll} 
Total admissions per day & 70.3 & 82.1 & $0.86(0.82-0.90)^{*}$ & 71.7 & 84.3 & $0.85(0.81-0.89)^{*}$ \\
\hline
\end{tabular}

Malignant neoplasms of respiratory and intrathoracic organs (ICD C30-C39) number of hospitals: 71, number of admissions: 11,016

$\begin{array}{lllllll}\text { Total admissions per day } & 55.3 & 63.1 & 0.88(0.83-0.92)^{*} & 51.5 & 64.5 & 0.80(0.76-0.84)^{*}\end{array}$

Malignant neoplasms of bone and articular cartilage and of mesothelial and soft tissue (ICD C40, C41, and C45-C49), number of hospitals: 58, number of admissions: 3,488

Total admissions per day

17.1

$19.9 \quad 0.86(0.78-0.94)^{*}$

18.3

18.9

$0.97(0.88-1.06), 0.50$

Melanoma and other malignant neoplasms of the skin (ICD C43 and C44), number of hospitals: 60, number of admissions: 8,383

$\begin{array}{lllllll}\text { Total admissions }=8,383 & 40.6 & 49.8 & 0.82(0.77-0.87)^{*} & 40.8 & 47.2 & 0.86(0.81-0.92)^{*}\end{array}$

Malignant neoplasms of the breast (ICD C50), number of hospitals: 69, number of admissions: 9,047

$\begin{array}{lllllll}\text { Total admissions per day } & 48.2 & 53.0 & 0.91(0.86-0.96)^{*} & 44.1 & 47.2 & 0.94(0.88-0.99)^{*}\end{array}$

Malignant neoplasms of the female genital organs (ICD C51-C58), number of hospitals: 67, number of admissions: 2,985

$\begin{array}{llllllll}\text { Total admissions per day } & 14.7 & 18.4 & 0.80(0.72-0.88)^{*} & 13.8 & 16.6 & 0.83(0.75-0.93)^{*}\end{array}$

Malignant neoplasms of the male genital organs (ICD C60-C63), number of hospitals: 65, number of admissions: 4,615

$\begin{array}{lllllll}\text { Total admissions per day } & 23.6 & 25.6 & 0.92(0.85-1.00) \text {, ns } & 22.6 & 26.3 & 0.86(0.79-0.93)^{*}\end{array}$

Malignant neoplasms of the urinary tract (ICD C64-C68), number of hospitals: 67, number of admissions: 5,621

$\begin{array}{lllllll}\text { Total admissions per day } & 29.1 & 32.1 & 0.91(0.84-0.98)^{*} & 27.6 & 30.8 & 0.89(0.83-0.96)^{*}\end{array}$

Malignant neoplasms, stated or presumed to be primary, of lymphoid, hematopoietic, and related tissues (ICD C81-C96), number of hospitals: 65, number of admissions: 9,200

$\begin{array}{lllllll}\text { Total admissions per day } & 46.7 & 56.2 & 0.83(0.79-0.88)^{*} & 41.6 & 51.2 & 0.81(0.77-0.86)^{*}\end{array}$

Asterisks denote significance. CI, confidence interval; ns, not significant.

pital (large full-service hospitals and small hospitals), and the number of COVID-19 cases in the federal states in which the hospitals are located.

In addition, we analyzed the interaction between different age groups, gender, hospital volume, and COVID-19 case volume during both periods, and found a significant difference in age, i.e., $\geq 75$ years versus $\leq 64$ years, so hospital admissions of older patients were more affected than those of younger patients. A significant interaction was seen for hospital volume and COVID-19 case volume, showing a more pronounced reduction in hospital admissions in both study cohorts in larger hospitals than in smaller hospitals, and also a higher impact in federal states with high COVID-19 case numbers than in those with lower numbers. Gender had no impact on admission numbers.

Table 2 shows the results for the different cancer diagnosis groups separately. Again, study cohorts were compared to the control cohorts in both time periods, respectively. In the early period, a significant difference was seen in all diagnostic groups, with the exception of malignant neoplasms of the male genital organs for which only a borderline significance was reached. In the later period, all incidence comparisons between study group and con- trol group were significant, with the exception of bone and soft tissue sarcomas, where no more difference is seen. This is likely due to the fact that the vast majority of these patients are seen in one large sarcoma referral center in Berlin. In total, with the exception of sarcomas, the expected recovery in the late period did not in fact take place.

\section{Discussion}

The lockdown due to the COVID-19 pandemic had a significant impact on hospital admissions for cancer diagnosis and treatment. Elderly patients were obviously more affected by the reduced number of hospital admissions than younger patients, probably because their personal health risk was considered especially high. In the federal states with higher numbers of COVID-19 cases, hospital admissions were reduced to a much greater extent than in states with lower numbers. The effect of hospital volume could well be a result of smaller numbers, rather than the assumption that small hospitals were not affected. A limitation of our analysis is, that university medicine is not sufficiently represented since there is 
only one university hospital in the Helios network. However, recent data from 310 hospitals within the IQM network have shown a pronounced reduction in numbers at all types of hospitals, i.e., university hospitals, community hospitals, and privately owned hospitals as well as at all sizes of hospital (maximum-care and basic-care hospitals). The effect was seen not only in elective treatments, where a delay is acceptable, but also in cancer care and emergency treatments like heart attack and stroke [5]. We therefore conclude that our analysis can be regarded as representative for Germany.

Reasons for the decline in hospital admissions are complex and require further investigation. Patients may have been reluctant to seek medical attention due to the fear of contagion at the hospital. In addition, the emphasis on social distancing might have inappropriately persuaded patients to avoid in-person medical care. Another reason may have been difficulty in getting appointments in outpatient care since many practices were either closed or had at least reduced their daily patient consultations.

A more detailed analysis of interventional treatments for heart failure and cardiac arrhythmias within the Helios hospital system showed a $13-28 \%$ decline during the COVID-19 outbreak. The impact of this decrease on long-term morbidity and mortality is currently unclear [6]. The UK recently published data on the impact of the COVID-19 pandemic on cancer deaths due to delays in diagnosis [7]. The different scenarios used in that study projected an increase in cancer deaths due to breast cancer, colorectal cancer, lung cancer, and esophageal cancer of around 9, 16, 5, and 6\%, respectively, within 5 years.

\section{Conclusions}

In contrast to the general concept to not limit medical care in the context of urgent cancer diagnosis and treatment, a statistically significant decrease in hospital ad- missions for cancer patients in the range of $10-20 \%$ was found for both study periods in comparison to the previous year controls. Obviously, the expected recovery did in fact not take place so early. The relative impact of reduced outpatient diagnoses, limited hospital resources, or patients' reservations and fears cannot yet be analyzed to explain this difference, but all of these factors together might have contributed to this significant effect. Also, the possible impact on prognosis and late outcome due to later diagnosis and treatment will only become evident in the future and should be analyzed accordingly.

\section{Statement of Ethics}

Only anonymized, aggregated routine data were used, so patient consent was not required and the paper was exempted from ethics committee approval.

\section{Conflict of Interest Statement}

P.R. received honoraria from Bayer, Clinigen, BMS, Roche, MSD, Deciphera, Novartis, Pfizer, PharmaMar, Lilly, Amgen. B.G. received honoraria from Roche, Celgene, Gilead, Janssen, Novartis, Riemser, MSD, Jazz, Amgen. M.U. received honoraria from Abbvie, Amgen, Astra Zeneca, Celgene, Clovis, Daichii Sankyo, Eisai, Lilly, MSD Merck, Mundipharma; Myriad Genetics, Novartis, Odonate, Pfizer, PUMA Biotechnology, Pierre Fabre, Roche Pharma, Sanofi Aventis. A.B., S.H., A.R., D.A., and R.K. have no conflicts of interest to declare.

\section{Funding Sources}

There are no funding sources to declare.

\section{Author Contributions}

All authors made substantial contributions to the conception of the work, analysis of the data, and writing of the manuscript as well as approving the final version.

\section{References}

1 Baayen RH, Davidson DJ, Bates DM. MixedEffects Modeling with Crossed Random Effects for Subjects and Items. J Mem Lang. 2008;59(4):390-412.

2 Kliegl R, Masson ME, Richter EM. A Linear Mixed Model Analysis of Masked Repetition Priming. Vis Cogn. 2010;18(5):655-81.

3 Bates D, Mächler M, Bolker B, Walker S. Fitting Linear Mixed-Effects Models Using Lme4. J Stat Softw. 2015;67(1):1-48.

4 R Core Team. R: A Language and Environment for Statistical Computing. 2019.
5 Kuhlen R, Schmithausen D, Winklmair C, Schick J, Scriba P. The effects of the COVID-19 pandemic and lockdown on routine hospital care for other illnesses. Dtsch Arztebl Int. 2020 Jul;117(27-28):488-9.

6 Bollmann A, Hohenstein S, Meier-Hellmann A, Kuhlen R, Hindricks G. Emergency hospital admissions and interventional treatments for heart failure and cardiac arrhythmias in Germany during the Covid-19 outbreak: insights from the German-wide Helios hospital network. Eur Heart J Qual Care Clin Outcomes. 2020 Jul;6(3):221-2.
7 Maringe C, Spicer J, Morris M, Purushotham A, Nolte E, Sullivan R, et al. The impact of the COVID-19 pandemic on cancer deaths due to delays in diagnosis in England, UK: a national, population-based, modelling study. Lancet Oncol. 2020 Aug;21(8):1023-34. 